\title{
THE FRACTAL TURBULENCE AND THE ORIGIN OF LARGE SCALE STRUCTURE
}

\author{
Y. - Z. Liu. Z. -G. Deng \\ Department of Physics. Graduate School \\ Academla Sinica \\ P. O. Box 3908 \\ Beljing. CHINA
}

\begin{abstract}
We have suggested a scenarlo of fractal turbulence which might explain the origin of galaxies and the observed large scale structure of the universe (Llu and Deng. 1987). Under the condition of the early universe, the cosmic fluid can be regarded as incompressible. If we assume that the density perturbations in the early universe are adlabatic and have the scale-free Zeldovich spectrum. We may obtain the spectrum of the velocity perturbations. Perturbations with scales less than horizon will undergo dissipative process by Thomson scattering. So. the cosmic fluid can be considered as a viscous fluid (Peebles. 1971). We can find the largest and smallest scale of the perturbations in the cosmic fluid by taking account of the Reynold's number on given scale and the scale of horizon. Using the present values of Hubble constant and the mean density of matter. we have found that on the scale of horizon the Reynold's number is just the order of $10^{2}$. This result shows that perturbations with scale a little smaller than horizon may produce Karman vortices before recombination and the vortices might form fractal turbulence due to Thomson drag.
\end{abstract}

From the scenario presented above we can estimate the characteristic mass and angular momentum of the elementary eddles which would be corresponding to the galaxies. The results are $M_{g} \simeq 10^{9}-10^{12}\left(\Omega h^{2}\right)^{-11 / 4} M_{0}$ and $J_{g} \simeq 10^{70}-10^{74}\left(\Omega h^{2}\right)^{-19 / 4} \mathrm{~g} \cdot \mathrm{cm}^{2} \cdot \mathrm{s}^{-1}$ which are colncident with the conventionally accepted values. It is also followed from this scenario that the large scale distribution of galaxies would likely to be a fractal sponge. We may infer that the fractal dimension of the large scale structure is about 1.25 which gives a power index of the correlation function in the distribution of galaxies to be about 1.75. These results are also coincident with observations (Peebles. 1980), and it is the first time to give a natural explanation for the power index of the two point correlation function of galaxies. Furthermore. from this scenario we could give the spectrum of volds with different scale in the large scale distribution.

Liu Y. -Z. and Deng Z. -G. . 1987. Ap.S.S. . in press.

Peobles. P.J.E. . 1971. Physlcal Cosmology (Princeton Univ. Press).

Peebles. P.J.E. . 1980. The large scale structure of the universe

(Princeton Univ. Press). 